Research Paper

\title{
MiR-34a-5p and miR-452-5p: The Novel Regulators of Pancreatic Endocrine Dysfunction in Diabetic Zucker Rats?
}

\author{
Tong $\mathrm{Su}^{1}$, Jiejun $\mathrm{Hou}^{2}$, Tonghua $\mathrm{Liu}^{3}$, Pei Dai ${ }^{1}$, LingLing Qin³ ${ }^{3}$ Lei Ding ${ }^{1}$, Yan Hu${ }^{1}$, Xiangyu Guo ${ }^{\circledR}$ \\ 1. Dongfang Hospital of Beijing University of Chinese Medicine, Beijing, Beijing 100078, China. \\ 2. Affiliated hospital of Shan'xi University of Chinese Medicine, Xianyang, Shanxi 712000, China. \\ 3. Beijing University of Chinese Medicine, Beijing, Beijing 100029, China \\ $\triangle$ Corresponding author: Xiangyu Guo Address: Dongfang Hospital of Beijing University of Chinese Medicine, Beijing, Beijing 100078, China. Telephone: \\ +8617710397258 E-mail address: gxyc1003@163.com
}

(C) The author(s). This is an open access article distributed under the terms of the Creative Commons Attribution License (https://creativecommons.org/licenses/by/4.0/). See http://ivyspring.com/terms for full terms and conditions.

Received: 2021.05.17; Accepted: 2021.06.30; Published: 2021.07.11

\begin{abstract}
Objective: The pancreatic endocrinal system dominates the regulation of blood glucose levels in vivo, and the dysfunction of pancreatic endocrine $\beta$-cells is a major cause of the occurrence and development of Type 2 diabetes (T2D). Although microRNA (miRNA) have been found to be key regulators of pancreatic $\beta$-cells proliferation, differentiation and apoptosis, the underlying mechanism remains enigmatic. The aim of this study was to identify several novel miRNAs which might be involved in the etiopathogenesis of diabetic $\beta$-cells dysfunction.

Methods: The miRNA expression profiles in the pancreas of high-fat diet (HFD) fed Zucker diabetic fatty (ZDF) rats and Zucker lean (ZL) rats feed with normal-fat diet (NFD) were detected by using miRNA microarray chip, and individually verified the most significant factors by quantitative real-time polymerase chain reaction (qRT-PCR) assay. The Gene Ontology (GO) and Kyoto Encyclopedia of Genes and Genomes (KEGG) enrichment analyses were used to predict the target genes related to each of the identified miRNAs and the functions of these target genes in different metabolic signaling pathways.

Results: Compared with the ZL rats, a total of 24 differentially expressed miRNAs were detected in ZDF rats. Among which miR-34a-5p and miR-452-5p were the most significantly up-regulated and down-regulated respectively. These miRNAs have not been reported in rats' pancreas before. By GO and KEGG enrichment analyses, we found that miR-34a-5p could negatively regulate pancreatic $\beta$-cell proliferation through the involvement of Wnt signaling pathway. In addition, it was also found to regulate insulin secretion through the insulin signaling pathway to modulate blood glucose levels. At the same time, miR-452-5p was found to positively regulate the activity of the key rate-limiting enzyme branched-chain $\alpha$-keto acid dehydrogenase- $\beta$ (BCKDHB) in the catabolism of branched chain amino acids (BCAA), leading to mitochondrial dysfunction in pancreatic $\beta$-cells.

Conclusions: miR-34a-5p and miR-452-5p were identified as the novel regulators of pancreatic endocrine dysfunction. These miRNAs might have the potential to be utilized as the new predictive biomarkers for the diagnosis of the occurrence and development of $T 2 \mathrm{D}$, as well as the therapeutic targets for T2D treatment.
\end{abstract}

Key words: Type 2 diabetes, Pancreatic endocrine dysfunction, miR-34a-5p, miR-452-5p, Biomarkers

\section{Introduction}

Insulin secreted by pancreatic endocrine $\beta$-cells is one of the major hormones that regulate glucose concentrations in the human body. Hence, dysregulation of insulin secretion is closely related to the occurrence and progression of type 2 diabetes (T2D). According to UK Prospective Diabetes Study (UKPDS)[1, 2], Homeostasis Model Assessment (HOMA) index of $\beta$-cell function (HOMA- $\beta$ ) indicates 
that $\beta$-cell function is reduced by $50 \%$ in T2D patients, and it exhibits progressive decline at a rate of approximately $5 \%$ per year. It is frustrating that the continuous loss of $\beta$-cell function and mass cannot be stopped and/or reversed by available therapeutic intervention regimens (dieting, exercise, or even hypoglycemic drugs). Therefore, the functional modulation of pancreatic $\beta$-cells has always been a central focus in diabetes research. Although apoptosis and dedifferentiation are considered to be the main causes of $\beta$-cell dysfunction leading to T2D, however, there are still many yet unexplored mechanisms, including modulation of $\beta$-cells' gene expression, RNA processing and maturation, as well as the activation of corresponding signal transduction pathways.

MicroRNA (miRNA) is a class of small non-coding RNA molecules with 21-23 nt in length[3]. Since the discovery of evolutionarily conserved pancreatic $\beta$ cell-specific miR-375, regulating insulin secretion, in 2004 by Matthew N. Poy et al.[4], a large number of miRNAs have been identified that are involved in the onset of diabetes. For example, Nadine Baroukh et al.[5] has reported that miR-124a plays a role in the formation of pancreatic cells. The primary function of these miRNAs includes facilitating the formation of an RNA-induced silencing complex (RISC) to regulate the stability of target mRNAs by binding to their 3'-untranslated region (UTR), thereby further controlling the protein levels of those particular target genes[6]. These functions of those miRNAs can directly affect $\beta$-cell proliferation, differentiation, maturation, and apoptosis[7]. Therefore, inhibiting or accelerating the expression of the regulatory miRNAs in pancreatic $\beta$-cells can be a new therapeutic approach to treat diabetic patients[8].

The genotype-phenotype interaction of glucose metabolism in T2D is very complex and greatly varies across the species[9]. Importantly, most current research studies on pancreatic miRNA profiles have been mainly conducted on mice and humans. Furthermore, there has been a lack of miRNAs expression profiling analyses of rats. In this study, we aimed to analyze the miRNA expression profiles in the pancreatic cells of the high-fat-diet (HFD) induced Zucker diabetic fatty $(\mathrm{ZDF}, \mathrm{fa} / \mathrm{fa})$ rats and the normal-fat-diet (NFD) fed Zucker lean (ZL, +/fa) rats using miRNA microarray chip technology, and single validated selected miRNAs using quantitative real-time polymerase chain reaction (qRT-PCR) to identify novel miRNAs which may be involved in the etiopathogenesis of pancreatic endocrine $\beta$-cell dysfunction of T2D.

\section{Materials and Methods}

\section{Animals and pancreatic tissues collection}

7-weeks-old male ZDF rats and ZL rats with weights ranging from $180 \mathrm{~g}$ to $200 \mathrm{~g}$, were purchased from Beijing Vital River Laboratory Animal Technologies (Beijing, China). All rats were housed in a specific pathogen-free facility in the Beijing University of Chinese Medicine. After one week of adaptive feeding with regular food, ZDF rats were fed a (HFD) as the experimental group $(n=5)$, and $Z L$ rats were fed a (NFD) as a control group $(n=5)$ for about 4 weeks. Body weight and fasting plasma glucose levels were measured routinely every week. The animal experiment protocol was approved by Beijing University of Chinese Medicine.

\section{Measurement of plasma insulin, triglycerides, cholesterol, and FFA}

Rats were fasted for $12 \mathrm{~h}$ before being sacrificed, and blood samples were then obtained from individual rats for the measurement of the concentrations of fasting plasma insulin, triglycerides, cholesterol, and free fatty acids (FFA). Specific ELISA kits (Wako Pure Chemical Industries, Osaka, Japan) were used to measure the concentrations of plasma insulin following manufacturer's protocol. The detection values of triglycerides, cholesterol, and FFA were read on an AU400 Biochemical Analyzer. Then the average was calculated from triplicate values for each of the parameters.

\section{Pancreatic tissues collection and immunofluorescence staining}

After the rats were sacrificed, part of the pancreatic tissues was quickly removed and placed in the refrigerator at $-80^{\circ} \mathrm{C}$ for a future test. The

other portion was fixed in $4 \%$ paraformaldehyde for $24 \mathrm{~h}$, then embedded in paraffin, sliced into $5 \mu \mathrm{m}$ sections, deparaffinized with conventional xylene and gradient alcohol method. After that, the sections were washed with $1 \times$ PBS and treated with $0.01 \mathrm{M}$ sodium citrate buffer and placed in a microwave oven for antigen retrieval. While they were being cooled, Triton X-100 was used to permeabilize the cell membrane for $15 \mathrm{~min}$ at room temperature. Then sections were blocked with $10 \%$ goat serum for $30 \mathrm{~min}$ at room temperature. Then the sections were added with the mixed primary antibodies [Insulin Antibody (1:100 dilution, Boster Biological Technology, USA), Glucagon Polyclonal Antibody (1:100 dilution, Bioss Antibodies, China), mixed in 1:1 ratio] and incubated overnight at $4{ }^{\circ} \mathrm{C}$. The next day, slides were placed for $1 \mathrm{~h}$ to restore room temperature, following staining with mixed fluorescent secondary antibodies 
[Fluorescein-Conjugated AffiniPure Goat Anti-Mouse IgG $(\mathrm{H}+\mathrm{L})$ (1:100 dilution, ZSGB-BIO, China), Fluorescein-Conjugated AffiniPure Goat Anti-Rabbit IgG (H+L) (1:100 dilution, ZSGB-BIO, China), mixed in 1:1 ratio] were added, the sections were then incubated at room temperature for $1 \mathrm{~h}$ in the dark, and the nucleus was subsequently stained with DAPI. At the final step, the fluorescence quenching agent was added, and sections were mounted with a cover slip. A fluorescence microscope was used to observe the results and capture the images.

\section{MicroRNA isolation and microarray analysis}

MiRNeasy Mini Kit (Qiagen, Germany) were used to extract total RNA from the pancreas tissue samples for comparison. First, the 5'end phosphate group of RNA was removed by using the dephosphorylation reaction mixture. After the sample denaturation and labeling reaction, the fluorescent dye was conjugated to the 3'end of RNA under the action of RNA ligase, then the hybridization reaction solution was added. After that, added samples and install on the Agilent's miRNA chip platform, according to the manufacturer's instructions. The hybridization was carried out at $55^{\circ} \mathrm{C}$ and $20 \mathrm{rpm}$ for 20h. After washing and scanning, the chips were scanned with Agilent Feature Extraction (AFE) software version 10.7.1.1, and the original data were normalized as suggested.

\section{Prediction and functional analysis of microRNA targets}

TargetScan (http://www.targetscan.org/) and miRDB (http://mirdb.org/miRDB/) were used for target gene prediction. For each set of predicted target genes, the function and pathway enrichment analyses were performed based on the Gene Ontology (GO, http://www.geneontology.org) and Kyoto Encyclopedia of Genes and Genomes (KEGG, http://www.kegg.jp/kegg/pathway.html) databases at the same time. All the data with $\mathrm{P}<0.05$ were considered statistically significant. More than 2 times of high expression or 0.5 times of low expression was considered highly significant.

\section{qRT-PCR}

Mircute miRNA isolation Kit (Tiangen, Beijing) was used to extract total RNA from pancreatic tissues. Then the total RNA was reversely transcribed into cDNA with GoScript ${ }^{\mathrm{TM}}$ Reverse Transcription System Kit (Promega, USA) according to the manufacturers' instructions. For cDNA preparation, $1 \mu \mathrm{g}$ of the total RNA was incubated at $70^{\circ} \mathrm{C}$ for $10 \mathrm{~min}$, and then the reaction tubes were kept on the ice after giving a short centrifugation. The thermal cycling profile for the reverse transcription reaction was as follows: $25^{\circ} \mathrm{C}$ for $10 \mathrm{~min}, 42^{\circ} \mathrm{C}$ for $1 \mathrm{~h}, 95^{\circ} \mathrm{C}$ for $5 \mathrm{~min}$, and $4^{\circ} \mathrm{C}$ for $5 \mathrm{~min}$. The relative levels of miR-34a-5p and miR-452-5p transcripts along with the internal control U6 small RNA were determined by qRT-PCR using GoTaq ${ }^{\circledR}$ qPCR Master Mix (Promega, USA) on the Applied Biosystems 7500 Real Time PCR System. The reaction conditions were as follow: $95^{\circ} \mathrm{C}$ for $2 \mathrm{~min}$ to pre-degeneration, then $95^{\circ} \mathrm{C}$ for $15 \mathrm{~s}, 60^{\circ} \mathrm{C}$ for $1 \mathrm{~min}$ and $72^{\circ} \mathrm{C}$ for $10 \mathrm{~min}$ loop 40 times. The primer sequences used in this qRT-PCR assay are listed in Table S3.

\section{Statistical analysis}

SPSS 22.0 software was used to analyze the data. All data are represented as mean \pm standard deviation (SD). The comparison of body weight, fasting glucose, plasma insulin, triglycerides, cholesterol, and FFA between two groups was performed using the Mann-Whitney U test, and the qRT-PCR data was performed with the unpaired student t-test method. Experimental results with $\mathrm{P}<0.05$ were considered statistically significant.

\section{Results}

\section{Effects of HFD-feed on ZDF rats}

Body weight and fasting blood glucose levels of the control and experimental rats were routinely measured every week to confirm that the average body weight of the ZDF rats was obviously higher than that of the control group rats $(\mathrm{P}<0.001$ of any week's data, Figure 1A, Table S1). The fasting blood glucose levels of the ZDF rats significantly increased and within diabetic range compared to that in control rats $(\mathrm{P}<0.001$ of any week's data, Figure $1 \mathrm{~B}$, Table S2). Our results showed that the levels of plasma insulin ( $9.87 \pm 1.05$ vs. $29.72 \pm 2.27 \mu \mathrm{U} / \mathrm{ml}, \mathrm{P}<0.001$, Figure $1 \mathrm{C}$ ), triglyceride $(1.97 \pm 0.08$ vs. $2.87 \pm 0.49 \mathrm{mmol} / \mathrm{l}, \mathrm{P}<0.01$, Figure 1D), cholesterol $(0.57 \pm 0.07$ vs. $4.86 \pm 1.52$ $\mathrm{mmol} / \mathrm{l}, \mathrm{P}<0.001$, Figure $1 \mathrm{E})$, and FFA content $(0.24 \pm 0.01$ vs. $0.58 \pm 0.03 \mathrm{mmol} / \mathrm{l}, \mathrm{P}<0.001$, Figure $1 \mathrm{~F})$ were significantly higher in $\mathrm{ZDF}$ rats compared to those in ZL rats.

\section{Diabetes-induced pathological changes of pancreas morphology}

First, we examined diabetes-induced morphological differences in pancreas between the control and diabetic rats by immunofluorescence (IF) staining with tissue-specific cell surface markers (Figure 2), which revealed that the pancreatic islets of normal ZL rats had regular morphology and clear boundaries, the a cells secreting glucagon were distributed uniformly around the islets, the population of $\beta$ cells was normal and distributed in the center of the islets, and the level of insulin secretion was higher (Figure 
2A). While the pancreatic islets of diabetic ZDF rats exhibited atrophy, with irregular tissue morphology and unclear borders, not only the number of $\beta$ cells was significantly reduced, but also the level of secreted insulin was insufficient along with the abnormal distribution of a cells and $\beta$ cells (Figure 2B).

\section{Cluster analysis of the differentially microRNAs in pancreas of ZDF rats}

Our cluster analysis of miRNA profiles showed that the expression levels of more than 700 miRNAs were altered in the pancreas of ZDF rats compared to that in ZL rats. Amongst the differentially expressed

A

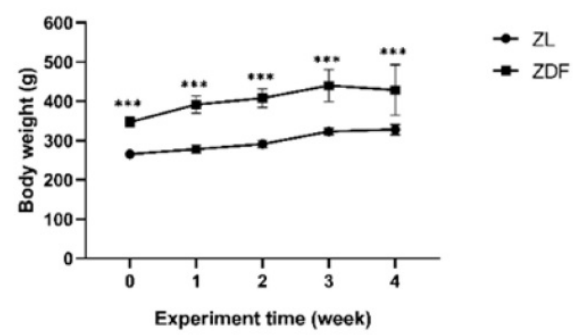

C

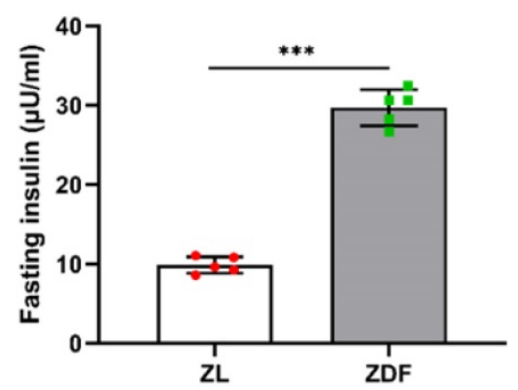

E

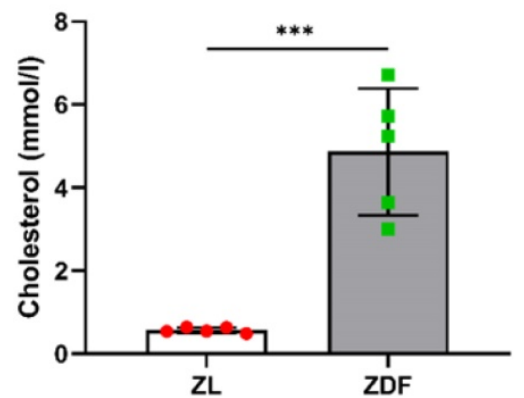

miRNAs, 10 miRNAs were highly expressed, while another 14 miRNAs exhibited significantly reduced expression levels, especially miR-34a-5p and miR-452-5p, which showed the most significant difference according to the heat map (Figure 3 and Table 1). These two miRNAs have also been previously reported in other diseases, such as acute ischemic stroke, diabetic kidney disease, and gestational diabetes, but there has been no report suggesting their roles in diabetic pancreatic islet dysfunction. Hence, it is crucial to identify the functional consequences of differential expression of these miRNAs in diabetes.

B

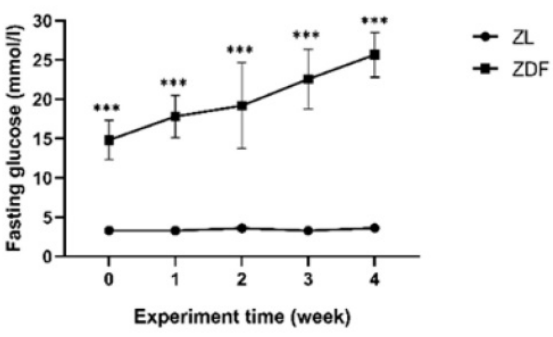

D

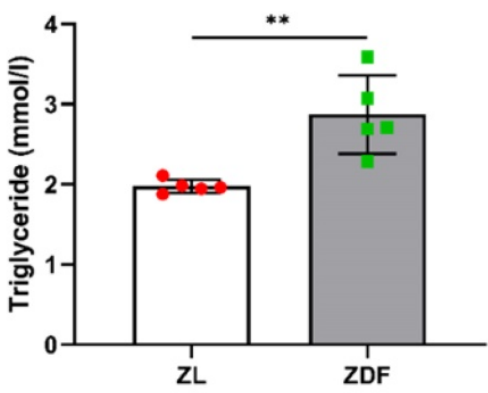

F

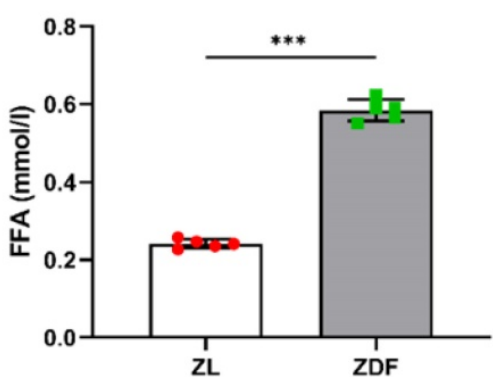

Figure 1. The effect of HFD-feed on ZDF rats. Rats' body weights (A) and fasting blood glucose (B) were measured on weekly basis. The levels of fasting plasma insulin (C), triglyceride (D), cholesterol (E) and FFA (F) were measured after 4 weeks feeding. The data of each group of rats $(n=5$ per group) are represented as mean \pm SD. Statistical significances between ZL and ZDF groups are indicated by ${ }^{*} \mathrm{P}<0.05$, ${ }^{* *} \mathrm{P}<0.01,{ }^{* * *} \mathrm{P}<0.001$. 

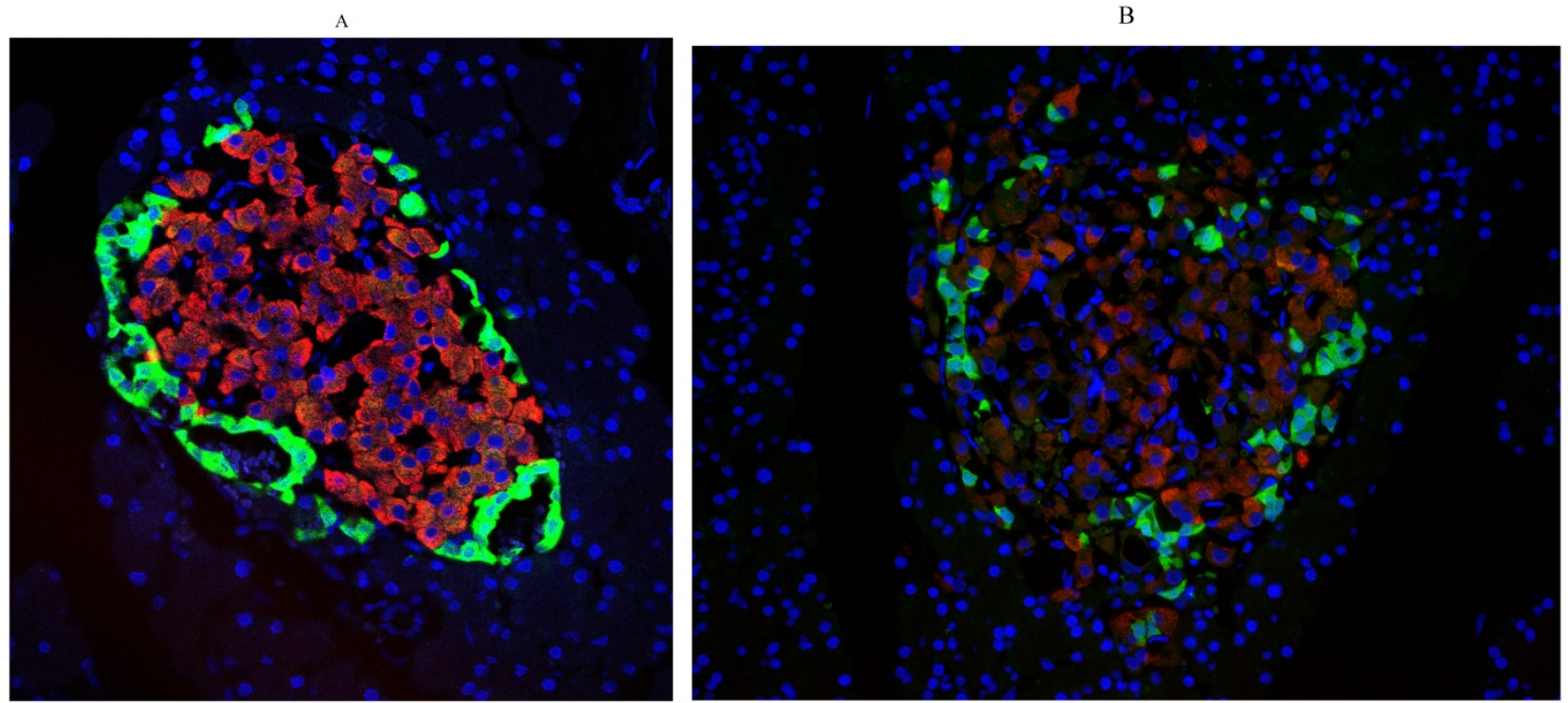

Figure 2. Pathological changes in the pancreas of the diabetic and normal rats. A) Represented the IF image of pancreatic islet cells of the control group. B) Represented IF image exhibiting the pathological changes in pancreatic islet cells in ZDF rats. Here, the red color indicated Insulin, green indicated Glucagon and blue indicated the DAPI staining of the nucleus. Scale bars, $20 \mu \mathrm{m} ; \mathrm{n}=5$ for each group.

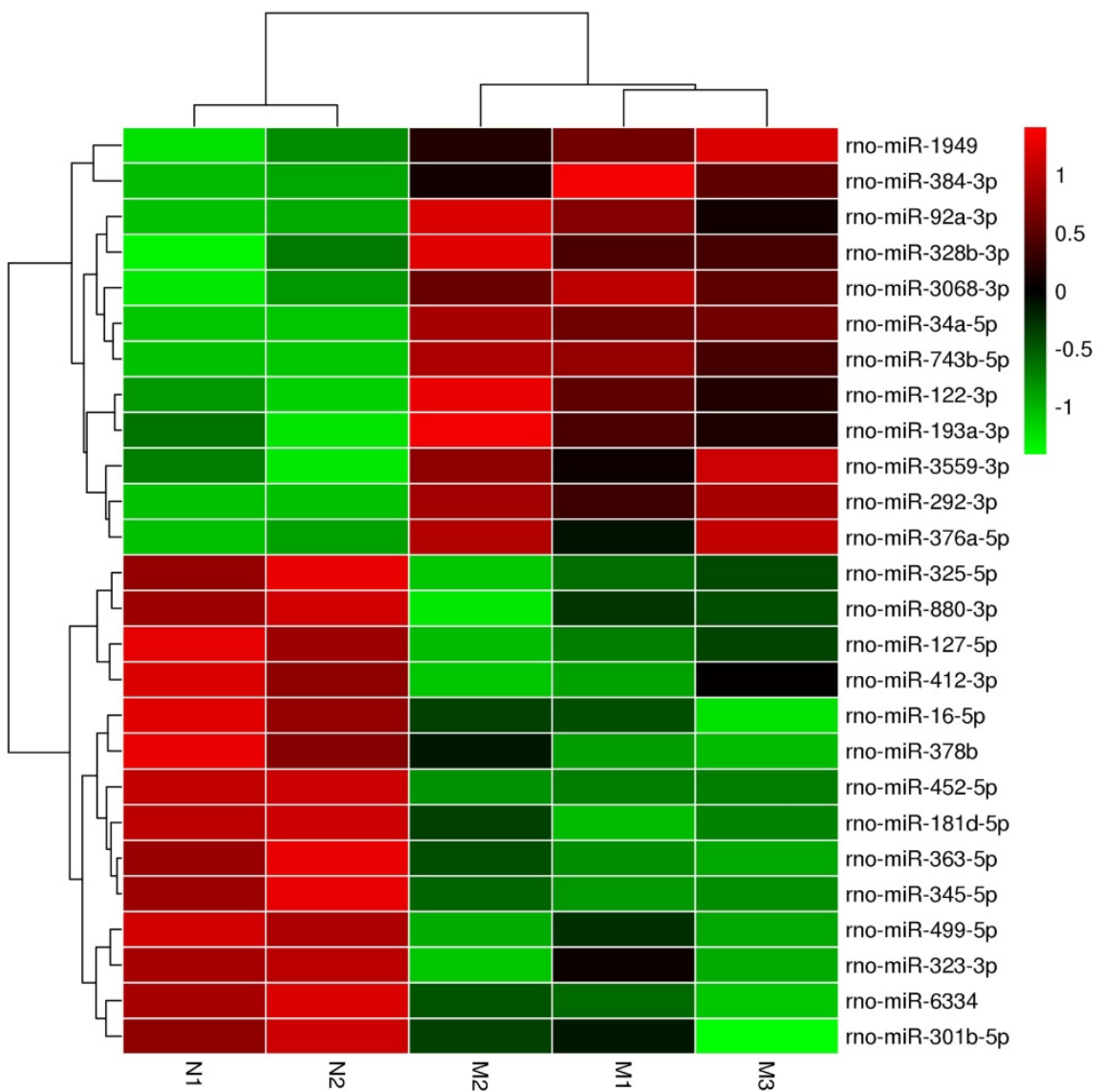

Figure 3. The heatmap analysis of differentially expressed miRNAs. In the cluster heatmap expression analysis, the red color indicated high expression level, green indicated low expression, and black indicated that there was no expression. The darker the color looks, the higher or lower the expression level is. Number N1 and Number N2 are the control (ZL) samples, number M1, Number M2 and NumberM3 are the experimental (ZDF) samples.

\section{GO and KEGG enrichment analysis}

The prediction of target genes of miR-34a-5p and miR-452-5p was based on the TargetScan and miRNA
Target Prediction Database (miRDB). The results showed that the miR-34a-5p with significantly high expression in the pancreas of ZDF rats had 332 target genes, while the miR-452-5p with significantly low 
expression had 341 target genes. Furthermore, GO analysis of miR-34a-5p target genes revealed that their primary function was to interact with steroid hormone receptors and $\beta$-catenin (Figure 4A), while the target genes of miR-34a-5p may functionally related to the synaptosomal-associated protein (SNAP) receptor activity (Figure 4C). Moreover, the KEGG analysis showed the involvement of miR-34a-5p in multiple crucial signaling pathways,

A

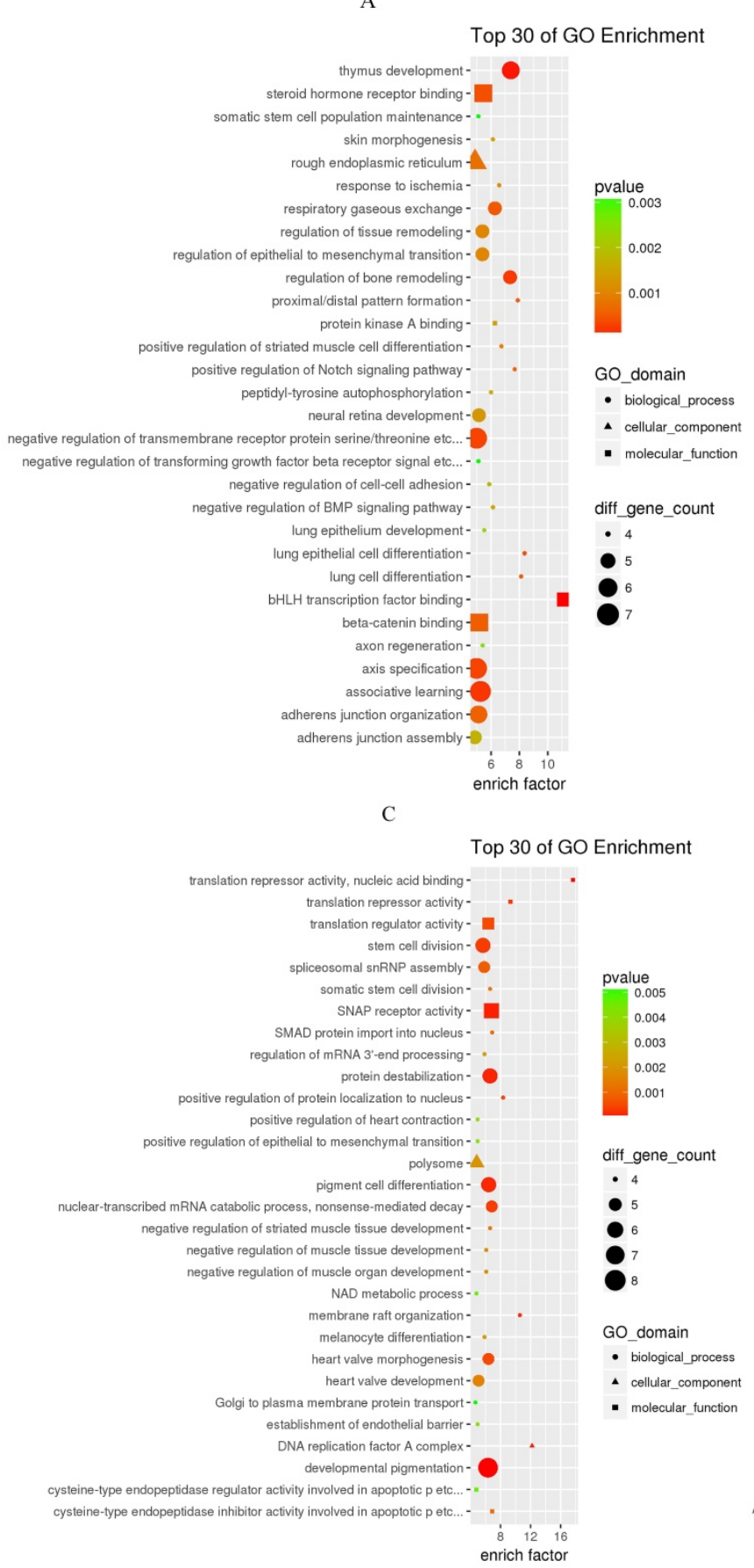

including Wnt signaling, gonadotropin-releasing hormone $(\mathrm{GnRH})$ signaling, insulin signaling, and ErbB signaling pathways (Figure 4B). On the other hand, miR-452-5p was found to be involved in certain amino acid (valine, leucine and isoleucine) degradation pathways as well as the ubiquitin-proteasome pathway (UPP) for proteolysis (Figure 4D).

B

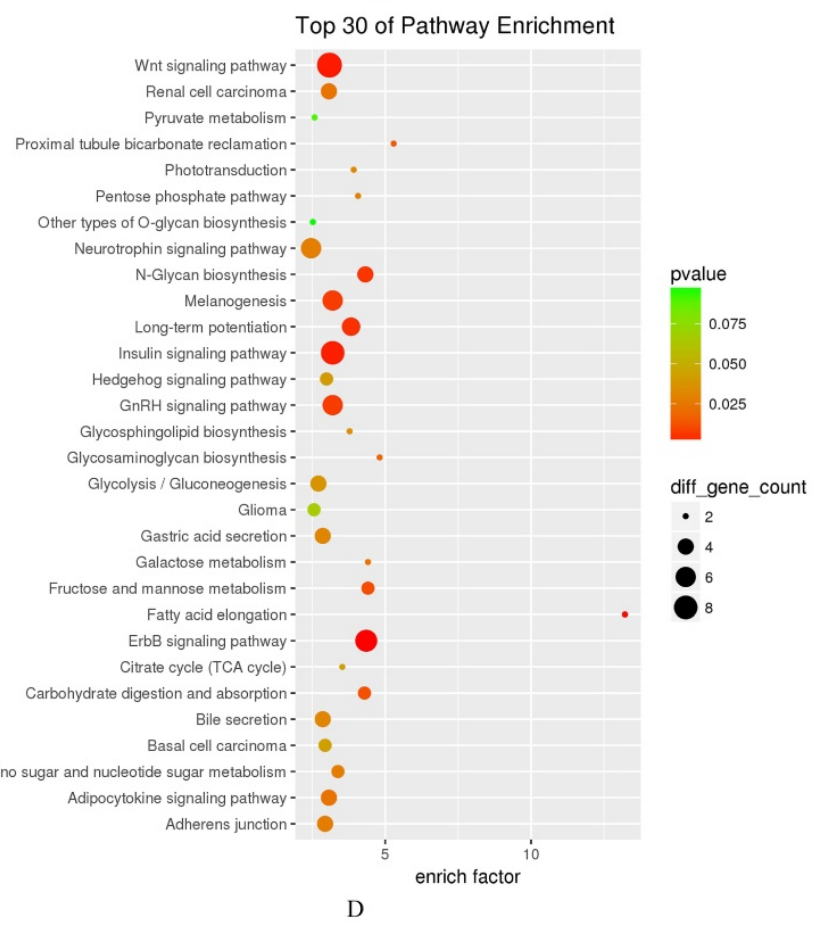

Top 30 of Pathway Enrichment

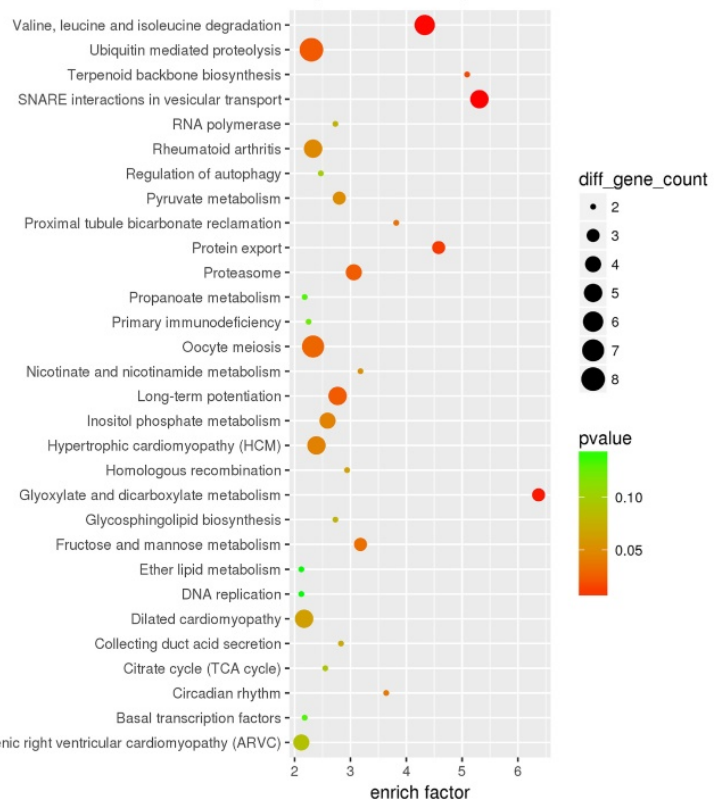

Figure 4. GO and KEGG enrichment analyses of the predicted target genes of miR-34a-5p and miR-452-5p. In the GO enrichment analysis figures (A and C), the circle indicated biological process, triangle indicated cellular component, and square indicated molecular function. The size of the graph indicated the number of different genes in the related item, the color indicated the significance of the difference, and the more to right of their position, the higher the level of enrichment. In the KEGG enrichment analysis figures (B and $\mathrm{D}$ ), the size of circles indicated the number of different genes in the related pathway, the color indicated the significance of the difference, and the more to right of their position, the higher the level of enrichment. 
Table 1. List of significant differentially expressed miRNAs in the pancreas.

\begin{tabular}{|c|c|c|c|c|c|}
\hline MicroRNA & $\mathrm{P}$-value & Fold Change & Nucleotide sequence & Genomic location & Mirbase accession_No \\
\hline \multicolumn{6}{|c|}{ Up-regulated microRNAs } \\
\hline rno-miR-34a-5p & 0.002903 & 2.188879 & ACAACCAGCTAAGACACTGC & chr5 & MIMAT0000815 \\
\hline rno-miR-743b-5p & 0.007534 & 1.032672 & ATGATGGACACCAGTCT & $\operatorname{chr} X$ & MIMAT0017288 \\
\hline rno-miR-292-3p & 0.012191 & 1.036416 & ACACTCAAAACCTGGCGGC & chr1 & MIMAT0000897 \\
\hline rno-miR-1949 & 0.021023 & 1.726167 & AACTATGCTGACATCCTG & $\operatorname{chr} 18$ & MIMAT0017852 \\
\hline rno-miR-3068-3p & 0.023419 & 1.102928 & TGTTGGAGTACTGCAATT & chr6 & MIMAT0024846 \\
\hline rno-miR-122-3p & 0.023779 & 1.034741 & TTAGTGTGATGATGGCG & $\operatorname{chr} 18$ & MIMAT0017116 \\
\hline rno-miR-92a-3p & 0.030293 & 1.471097 & CAGGCCGGGACAAGTGC & $\operatorname{chr} X$ & MIMAT0000816 \\
\hline rno-miR-3559-3p & 0.035091 & 1.050094 & CACGACAGACTCAGTA & $\operatorname{chr} X$ & MIMAT0017828 \\
\hline rno-miR-376a-5p & 0.043960 & 1.079268 & CTCATAGAAGGAGAATCTACC & chr6 & MIMAT0003197 \\
\hline rno-miR-384-3p & 0.045926 & 1.023334 & ATTGTGAACAATTTCTAGGAAT & $\operatorname{chr} X$ & MIMAT0005310 \\
\hline \multicolumn{6}{|c|}{ Down-regulated microRNAs } \\
\hline rno-miR-452-5p & 0.000039 & 0.017976 & CTCAGTTTCСTCTGCA & NA & MIMAT0035748 \\
\hline rno-miR-345-5p & 0.049379 & 0.483844 & GCACTGGACTAGGGGT & chr6 & MIMAT0000594 \\
\hline rno-miR-16-5p & 0.017979 & 0.586664 & CGCCAATATTTACGTGCTG & chr2 & MIMAT0000785 \\
\hline rno-miR-181d-5p & 0.009535 & 0.631897 & ACCCACCGACAACAATG & chr19 & MIMAT0005299 \\
\hline rno-miR-499-5p & 0.008271 & 0.904235 & AAACATCACTGCAAGTCTT & $\operatorname{chr} 3$ & MIMAT0003381 \\
\hline rno-miR-378b & 0.029414 & 0.910314 & ССТTСТGАСТССАА & $\operatorname{chr} 5$ & MIMAT0024855 \\
\hline rno-miR-325-5p & 0.019239 & 0.929482 & ACACTTACTGAGCACCTA & $\operatorname{chr} X$ & MIMAT0000557 \\
\hline rno-miR-127-5p & 0.012196 & 0.944294 & AATCAGAGCCCTCTGAG & chr6 & MIMAT0017117 \\
\hline rno-miR-880-3p & 0.019889 & 0.947277 & TCTACTCAGAATGAATGGAGT & $\operatorname{chr} X$ & MIMAT0005288 \\
\hline rno-miR-323-3p & 0.043819 & 0.972565 & AGAGGTCGACCGTGTAATGT & chr6 & MIMAT0000550 \\
\hline rno-miR-363-5p & 0.028662 & 0.973161 & AAATTGCATCGTGATCCAC & $\operatorname{chr} X$ & MIMAT0003209 \\
\hline rno-miR-412-3p & 0.026106 & 0.974151 & ACGGCTAGTGGACCAG & chr6 & MIMAT0003124 \\
\hline rno-miR-6334 & 0.005202 & 0.980032 & GCCGGCAGCTGG & $\operatorname{chr} 3$ & MIMAT0025075 \\
\hline rno-miR-301b-5p & 0.042853 & 0.985857 & AGTAGTGCAACCTAGTCA & chr11 & MIMAT0017300 \\
\hline
\end{tabular}

There are 6 parameters in the table, including P-value, fold change, nucleotide sequence, chromosome location and miRBase number. MiRNA expression difference was considered significant when $\mathrm{P}<0.05$, and fold change $\leq 0.5$ or fold change $\geq 2$.

A

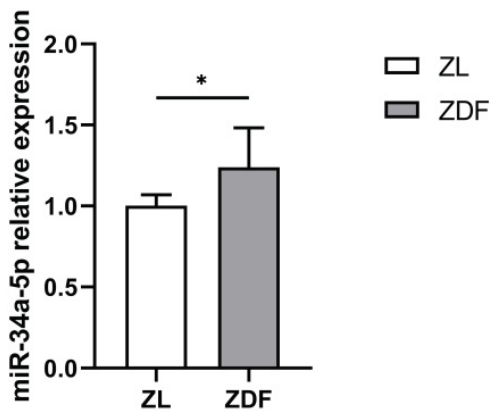

B

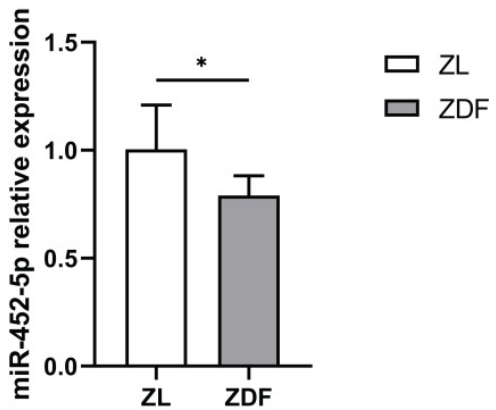

Figure 5. qRT-PCR analysis of miR-34a-5p and miR-452-5p expressions. Compared to $Z L$ rats in the control group, the expression of miR-34a-5p in the pancreas of diabetic ZDF rats was up-regulated (A), while miR-452-5p expression was down-regulated (B). The data shown are representative of at least three independent experiments ( $\mathrm{n}=4$ for each group, 3 replicate holes for each sample) and represented as mean $\pm S D$. The statistically significant is indicated by ${ }^{*} P<0.05$.

\section{The target genes of miR-34a-5p and miR-452-5p}

According to the KEGG analysis results, a total of 4 signaling pathways were mainly related to miR-34a-5p expression, and they corresponded to a total of 21 target genes. Meanwhile, there were 2 signaling pathways primarily related to miR-452-5p expression, and they corresponded to 14 target genes (Table 2).

Table 2. The pathway and target genes of miR-34a-5p and miR-452-5p

\begin{tabular}{|c|c|c|c|}
\hline PathwayID & Pathway Description & P-value & Target Genes \\
\hline \multicolumn{4}{|l|}{ miR-34a-5p } \\
\hline rno04310 & $\begin{array}{l}\text { Wnt signaling } \\
\text { pathway }\end{array}$ & 0.002230 & $\begin{array}{l}\text { DAAM2 MAPK10 PLCB2 PPP2CA } \\
\text { TCF7L1 CAMK2B WNT7A WNT1 } \\
\text { CAMK2A }\end{array}$ \\
\hline rno04910 & $\begin{array}{l}\text { Insulin signaling } \\
\text { pathway }\end{array}$ & 0.002749 & $\begin{array}{l}\text { MAPK10 PPP1CC CRKL PRKAG1 } \\
\text { LIPE HK1 PCK1 MKNK1 }\end{array}$ \\
\hline rno04912 & $\begin{array}{l}\text { GnRH signaling } \\
\text { pathway }\end{array}$ & 0.007240 & $\begin{array}{l}\text { MAP2K3 FSHB MAPK10 PLCB2 } \\
\text { CAMK2B CAMK2A }\end{array}$ \\
\hline rno04012 & $\begin{array}{l}\text { ErbB signaling } \\
\text { pathway }\end{array}$ & 0.000649 & $\begin{array}{l}\text { MAPK10 CRKL PAK6 CAMK2B } \\
\text { TGFA ABL1 CAMK2A }\end{array}$ \\
\hline \multicolumn{4}{|c|}{ miR-452-5p } \\
\hline rno00280 & $\begin{array}{l}\text { Valine, leucine and } \\
\text { isoleucine degradation }\end{array}$ & 0.001316 & $\begin{array}{l}\text { BCKDHB ALDH6A1 ACAT1 } \\
\text { ACADSB DBT MCCC2 }\end{array}$ \\
\hline rno04120 & $\begin{array}{l}\text { Ubiquitin mediated } \\
\text { proteolysis }\end{array}$ & 0.021330 & $\begin{array}{l}\text { UBA3 PRPF19 SIAH1 ANAPC11 } \\
\text { UBE2G1 UBA6 UBE2D1 XIAP }\end{array}$ \\
\hline
\end{tabular}

The expression of $\mathrm{miR}-34 \mathrm{a}-5 \mathrm{p}$ and $\mathrm{miR}-452-5 p$ in control vs. diabetic rat model

The qRT-PCR assay revealed that the expression of miR-34a-5p was up-regulated (Figure 
$5 \mathrm{~A})$, while that of miR-452-5p was down-regulated in the pancreas of the ZDF rats compared to those in control (ZL) rats (Figure 5B).

\section{Discussion}

Insulin is one of the critical hormones that regulate blood glucose homeostasis. The major physiological effects of insulin secretion include inhibition of hepatic gluconeogenesis and glycogenolysis, stimulation of skeletal glucose uptake, and suppression of adipose lipolysis[10]. These functions make pancreatic $\beta$-cells irreplaceable in the process of glucose metabolism. Once there is dysregulation of these physiological functions, such as delayed or decreased insulin secretion, hyperglycemia occurs.

Pancreatic endocrine $\beta$-cells function is affected by both genetics and/or environmental factors, including race, family history, age, disease course, and body mass index (BMI), etc.[11]. Patients with poor residual $\beta$-cell function are often required to administer exogenous insulin in the early stage of clinical treatment[12]. However, exogenous insulin treatment is only a mitigation strategy, since the protective effects disappear when there is no external insulin input. Also, other therapeutic medications, such as metformin[13], dipeptidyl peptidase-IV (DPP-4) inhibitors[14], glucagon-like peptide-1 (GLP-1) receptor agonists[15] and sodium glucose cotransporter-2 (SGLT2) inhibitors[16], have the protective effects on $\beta$-cells function in the short term, including improving insulin sensitivity, inducing $\beta$-cells' and inhibit a-cells' proliferation, but their long-term efficacy remains unclear. Therefore, the research on the mechanism of pancreatic endocrine $\beta$-cells' dysfunction is important.

The primary reason for the $\beta$-cell deficiency in the diabetic condition could be apoptosis and dedifferentiation[17]. However, the underlying molecular mechanisms of these deregulations have not been fully explored. MiRNAs are ubiquitously present in the eukaryotic cells, where they exercise a wide range of physiological functions. In pancreatic $\beta$-cells, miRNA maintains the balance among cell proliferation, differentiation and apoptosis[18]. When some miRNAs are overexpressed, they can induce dedifferentiation by inhibiting the expression of genes that maintain the mature phenotype of $\beta$-cells[19], or induce $\beta$-cells apoptosis by up-regulating the expression of pro-apoptotic genes[20], which eventually leads to diabetes.

In previous studies on miRNA profiling in rodents, most of the miRNA expression profiles were generated from peripheral blood[21], liver and other tissues[22]. In contrast, there are only a few miRNA expression profiles generated from pancreatic tissue samples. This may be because the pancreas secretes a large number of digestive enzymes, and those enzymes exert a stronger chemical degradation effect on the pancreatic tissue, making it difficult to extract a complete and intact miRNA expression profiles from these kinds of specimens.

ZDF rat model is a spontaneous well-established diabetic model and an ideal model for observing the natural progression of T2D[23]. ZDF rats have leptin receptor defects, which makes them exhibit the characteristics of metabolic syndrome from the early stages of development, such as obesity, hyperglycemia, and hyperinsulinemia, and eventually $\beta$-cell dysfunction[24, 25]. In this study, we fed ZDF rats with HFD for 4 weeks to detect the pancreatic differential miRNA expression-related genes in ZDF $(\mathrm{fa} / \mathrm{fa})$ rats and $\mathrm{ZL}(\mathrm{fa} /+)$ rats by microarray assays. Finally, among 24 candidate miRNAs, we found two miRNAs, miR-34a-5p and miR-452-5p, which showed the most significant regulatory effects on their target genes. These two miRNAs have previously been reportedly found in the lung[26-28], liver[28, 29], kidney[30, 31], and a certain type of cancer cells[32, 33]. In recent studies, miR-34a-5p has been found to mediate the lipotoxic effect of pancreatic $\beta$-cells in hyperlipidemia mice[34], while miR-452-5p has been reported to be involved in the tumorigenesis especially reproductive endocrine tumors such as prostate cancer[35] and breast cancer[36]. However, miR-34a-5p and miR-452-5p expressions have never been reported in the pancreas of diabetic individuals or animal models, and there is only a handful of research investigating their relationship with blood glucose metabolism. Therefore, we examined their target genes by using GO and KEGG enrichment analyses, and further verified their expressions by qRT-PCR assay.

In the KEGG enrichment analysis of miR-34a-5p target genes, we found that the Wnt and the insulin signaling pathways-related genes were the most enriched. The GO enrichment analysis also showed that the most important molecular function of miR-34a-5p was binding with $\beta$-catenin. The above results further confirmed that miR-34a-5p was closely related to the Wnt/ $\beta$-catenin signaling axis, one of the most critical links in the classic Wnt signaling pathway.

Wnt signaling pathway can affect $\beta$-cells proliferation[37]. Wnt protein is a highly conserved secreted protein. Its ligands bind and activate Frizzleds (Fz), a transmembrane receptor protein, thereby stabilizing $\beta$-catenin combined with transcription factor 7-like 2 gene (TCF7L2) and causing its nuclear translocation[38]. Dessimoz J et 
al.[39] has reported that selective inactivation of $\beta$-catenin results in $\beta$-cells' hypoplasia. Struan F A Grant et al.[40] reported that TCF7L2 plays an important physiological role both in pancreas and insulin sensitive organs, regulating the intestinal secretion of GLP-1 to induce $\beta$-cell proliferation[41].

Based on the above results, we speculate that miR-34a-5p may serve as an antagonist to $\beta$-catenin expression, blocking the conduction of Wnt signaling cascades in the pancreas, and inhibiting the expression of a variety of cell cycle regulators and functional transcription factors in $\beta$-cells. miR-34a-5p affects the development and functional maturity of $\beta$-cells, which in turn decreases the body's tolerance to glucose level and the insulin secretion. Meanwhile, the reduced level of $\beta$-catenin down-regulates the expression of TCF7L2, affecting the expression of the proglucagon gene in the ileum, thereby reducing the secretion of GLP-1, leading to T2D onset. At the same time, we found that Wnt1 and TCF7L1, the direct target genes of miR-34a-5p, were also involved in the conduction of the Wnt signaling pathway (Table 2). TCF7L1 is a Wnt inhibitor and stem cell regulator that affects the development of embryonic stem cells[42], suggesting that miR-34a-5p may affect the development of fetal stem cells of pancreatic lineage.

Another major pathway that miR-34a-5p is involved in is insulin signaling pathway which has the most direct regulatory effect on blood glucose homeostasis. Insulin signaling pathway mainly consists of the PI3K/Akt pathway and the ERK/MAPK pathway. The latter is a mitotic regulation pathway that causes cells proliferation and differentiation by activating specific transcription factors[43]. We found that the miR-34a-5p target gene related to the insulin signaling pathway was MAPK10. This suggests that miR-34a-5p may directly inhibit the expression of MAPK10 and reduce the activation of downstream transcription factors, thus affecting the proliferation and differentiation of $\beta$-cells.

miR-452-5p is the most down-regulated microRNA in the pancreas of the diabetic ZDF rats. The results of KEGG showed that its target genes were mostly enriched in valine, leucine, and isoleucine degradation pathway. Valine, leucine, and isoleucine all have functional $\mathrm{R}$ group branches, therefore, they are referred to as branched chain amino acids (BCAA)[44]. They are important nutrients and metabolic regulators that are beneficial to health. However, many studies have found that BCAAs play an important role in the pathogenesis of metabolic diseases in recent years[45, 46].

Since BCAAs are essential amino acids that can only be supplemented by food, their levels in the body can only be controlled through catabolism[47]. The most important key rate-limiting enzyme in this process is branched-chain a-keto acid dehydrogenase $(\mathrm{BCKDH})[48]$, if the activity of $\mathrm{BCKDH}$ reduced, the rate of the entire reaction system will decreases, leading to the suppression of BCAA catabolism, which will then lead to the accumulation of excessive BCAAs in the body that cannot be fully eliminated, thus building up the concentration of toxic metabolites. This serious result will further leads to the mitochondrial dysfunction in $\beta$-cells, and ultimately leading to T2D pathology[49]. The KEGG results showed that $\mathrm{BCKDHB}$ was a target gene of $\mathrm{miR}-452-5 \mathrm{p}$, and it has been indicated that miR-452-5p directly regulates the expression of $\mathrm{BCKDHB}$ gene, one of the major catalytic subunits of BCKDH. It has been further verified that the activities of $\mathrm{BCKDH}$ and its catalytic subunits all decrease under diabetic conditions[50]. In view of the low expression of miR-452-5p in the pancreas of ZDF rats, we are assuming that there might be a positive regulation between miR-452-5p and BCKDHB. The low expression of miR-452-5p might have inhibited the expression of the $\mathrm{BCKDHB}$ gene, leading to the accumulation of BCAAs in the pancreas and ultimately damaging $\beta$-cells.

In summary, there are still many miRNAs related to pancreatic endocrine $\beta$-cell dysfunction that have not yet been determined. Their functions and action mechanisms are being discovered with the development of scientific research. Therefore, we believed that our research of miR-34a-5p and miR-452-5p would be valuable in the light of understanding the miRNA-mediated regulation of T2D onset and progression.

However, there is a limitation associated with our study. We only conducted a preliminary study on these two miRNAs in vivo, and have not performed their functional verification like inhibition or overexpression. Hence, it is worth further investigating the functional implication of these miRNA in relation to diabetes in the future.

\section{Conclusion}

We identified that miR-34a-5p and miR-452-5p were significantly differentally expressed in the pancreas of ZDF rats. We speculated that they might be the novel miRNAs involved in the etiopathogenesis of pancreatic endocrine $\beta$-cells dysfunction, and might have the potential to be new predictive biomarkers related to T2D development and progression, as well as the therapeutic targets for T2D treatment. 


\section{Abbreviations}

T2D: Type 2 diabetes; miRNA: microRNA; ZDF: Zucker diabetic fatty; ZL: Zucker lean; HFD: high-fat diet; NFD: normal-fat diet; qRT-PCR: quantitative real-time polymerase chain reaction; GO: Gene Ontology; KEGG: Kyoto Encyclopedia Genes and Genomes; BCAA: branched chain amino acids; BCKDHB: branched-chain a-keto acid dehydrogenase- $\beta$; RISC: RNA-induced silencing complex; FFA: free fatty acids; TCF7L2: transcription factor 7-like 2 gene; TCF7L1: transcription factor 7-like 1 gene; DPP-4: dipeptidyl peptidase IV; GLP-1: glucagon-like peptide-1; SGLT2: Sodium glucose cotransporter 2; MAPK: mitogen-activated protein kinase; PI3K: phosphatidylinositol 3-kinase; Akt: protein kinase B; ERK: Extracellular signal-regulated kinase.

\section{Supplementary Material}

Supplementary tables.

http://www.medsci.org/v18p3171s1.pdf

\section{Acknowledgments}

This study was supported in part by research grants from of Project of Distinguished Young Scholars of Beijing University of Chinese Medicine (BUCM-2019-JCRC2009) and the National Natural Science Foundation of China (81303130).

\section{Author Contributions}

Tong Su: carried out experiments, wrote and edited the original draft, Xiangyu Guo: designed the experiments, reviewed, modified and edited the manuscript, Jiejun Hou and Tonghua Liu: conceived of the study, participated in its design and coordination, Pei Dai and Lei Ding: carried out experiments, LingLing Qin and Yan Hu: analyzed data. All authors read and approved this final manuscript.

\section{Competing Interests}

The authors have declared that no competing interest exists.

\section{References}

1. U.K. prospective diabetes study 16 . Overview of 6 years' therapy of type II diabetes: a progressive disease. U.K. Prospective Diabetes Study Group. Diabetes. 1995; 44: 1249-58.

2. Gannon NP, Schnuck JK, Vaughan RA. BCAA Metabolism and Insulin Sensitivity - Dysregulated by Metabolic Status? Molecular nutrition \& food research. 2018; 62: e1700756.

3. Mohr AM, Mott JL. Overview of microRNA biology. Seminars in liver disease. 2015; 35: 3-11.

4. Poy MN, Eliasson L, Krutzfeldt J, Kuwajima S, Ma X, Macdonald PE, et al. A pancreatic islet-specific microRNA regulates insulin secretion. Nature. 2004; 432: 226-30.

5. Baroukh N, Ravier MA, Loder MK, Hill EV, Bounacer A, Scharfmann R, et al. MicroRNA-124a regulates Foxa2 expression and intracellular signaling in pancreatic beta-cell lines. The Journal of biological chemistry. 2007; 282: $19575-88$
6. Kawamata T, Tomari Y. Making RISC. Trends in biochemical sciences. 2010; 35: 368-76

7. Eliasson L, Regazzi R. Micro(RNA) Management and Mismanagement of the Islet. Journal of molecular biology. 2020; 432: 1419-28.

8. Regazzi R. MicroRNAs as therapeutic targets for the treatment of diabetes mellitus and its complications. Expert opinion on therapeutic targets. 2018; 22: 153-60.

9. Chandrasekera PC, Pippin JJ. Of rodents and men: species-specific glucose regulation and type 2 diabetes research. Altex. 2014; 31: 157-76.

10. Petersen MC, Shulman GI. Mechanisms of Insulin Action and Insulin Resistance. Physiological reviews. 2018; 98: 2133-223.

11. Ashcroft FM, Rorsman P. Diabetes mellitus and the $\beta$ cell: the last ten years. Cell. 2012; 148: 1160-71.

12. Ahmad K. Insulin sources and types: a review of insulin in terms of its mode on diabetes mellitus. Journal of traditional Chinese medicine $=$ Chung i tsa chih ying wen pan. 2014; 34: 234-7.

13. Del Prato S, Bianchi C, Marchetti P. beta-cell function and anti-diabetic pharmacotherapy. Diabetes/metabolism research and reviews. 2007; 23: 518-27.

14. Ahrén B, Foley JE. Improved glucose regulation in type 2 diabetic patients with DPP-4 inhibitors: focus on alpha and beta cell function and lipid metabolism. Diabetologia. 2016; 59: 907-17.

15. Egan JM, Bulotta A, Hui H, Perfetti R. GLP-1 receptor agonists are growth and differentiation factors for pancreatic islet beta cells. Diabetes/metabolism research and reviews. 2003; 19: 115-23.

16. Asahara SI, Ogawa W. SGLT2 inhibitors and protection against pancreatic beta cell failure. Diabetology international. 2019; 10: 1-2.

17. Remedi MS, Emfinger C. Pancreatic $\beta$-cell identity in diabetes. Diabetes, obesity \& metabolism. 2016; 18 Suppl 1: 110-6.

18. Soltani A, Jafarian A, Allameh A. The Predominant microRNAs in $\beta$-cell Clusters for Insulin Regulation and Diabetic Control. Current drug targets. 2020; 21: 722-34.

19. Sebastiani G, Grieco GE, Brusco N, Ventriglia G, Formichi C, Marselli L, et al. MicroRNA Expression Analysis of In Vitro Dedifferentiated Human Pancreatic Islet Cells Reveals the Activation of the Pluripotency-Related MicroRNA Cluster miR-302s. International journal of molecular sciences. 2018; 19.

20. Belgardt BF, Ahmed K, Spranger M, Latreille M, Denzler R, Kondratiuk N, et al. The microRNA-200 family regulates pancreatic beta cell survival in type 2 diabetes. Nature medicine. 2015; 21: 619-27.

21. Juzenas S, Venkatesh G, Hübenthal M, Hoeppner MP, Du ZG, Paulsen M, et al. A comprehensive, cell specific microRNA catalogue of human peripheral blood. Nucleic acids research. 2017; 45: 9290-301.

22. Herrera BM, Lockstone HE, Taylor JM, Ria M, Barrett A, Collins S, et al. Global microRNA expression profiles in insulin target tissues in a spontaneous rat model of type 2 diabetes. Diabetologia. 2010; 53: 1099-109.

23. Delic D, Eisele C, Schmid R, Luippold G, Mayoux E, Grempler R. Characterization of Micro-RNA Changes during the Progression of Type 2 Diabetes in Zucker Diabetic Fatty Rats. International journal of molecular sciences. 2016; 17

24. Islam MS, Wilson RD. Experimentally induced rodent models of type 2 diabetes. Methods in molecular biology (Clifton, NJ). 2012 : 933: 161-74.

25. Shafrir E, Ziv E. A useful list of spontaneously arising animal models of obesity and diabetes. American journal of physiology Endocrinology and metabolism. 2009; 296: E1450-2

26. Shah D, Das P, Alam MA, Mahajan N, Romero F, Shahid M, et al. MicroRNA-34a Promotes Endothelial Dysfunction and Mitochondrial-mediated Apoptosis in Murine Models of Acute Lung Injury. American journal of respiratory cell and molecular biology. 2019; 60: 465-77.

27. Palleschi A, Gaudioso G, Edefonti V, Musso V, Terrasi A, Ambrogi F, et al. Bronchoalveolar Lavage-microRNAs Are Potential Novel Biomarkers of Outcome After Lung Transplantation. Transplantation direct. 2020; 6: e547.

28. Feili X, Wu S, Ye W, Tu J, Lou L. MicroRNA-34a-5p inhibits liver fibrosis by regulating TGF- $\beta 1 / \mathrm{Smad} 3$ pathway in hepatic stellate cells. Cell biology international. 2018; 42: 1370-6.

29. Garcia Garcia de Paredes A, Manicardi N, Tellez L, Ibañez L, Royo F, Bermejo J, et al. Molecular Profiling of Decompensated Cirrhosis by a Novel MicroRNA Signature. Hepatology communications. 2021; 5: 309-22.

30. Wang X, Chang C, Jin W, Arun A, Sudunabuqi S, Aodaofu A, et al. Identifying Key MicroRNAs Targeted by Narenmandula in a Rodent Nephropathy Model. Evidence-based complementary and alternative medicine : eCAM. 2020; 2020: 9196379.

31. Xie C, Wu W, Tang A, Luo N, Tan Y. IncRNA GAS5/miR-452-5p Reduces Oxidative Stress and Pyroptosis of High-Glucose-Stimulated Renal Tubular Cells. Diabetes, metabolic syndrome and obesity : targets and therapy. 2019; 12. 2609-17.

32. Li YY, Tao YW, Gao S, Li P, Zheng JM, Zhang SE, et al. Cancer-associated fibroblasts contribute to oral cancer cells proliferation and metastasis via exosome-mediated paracrine miR-34a-5p. EBioMedicine. 2018; 36: 209-20.

33. Warnecke-Eberz U, Chon SH, Hölscher AH, Drebber U, Bollschweiler E. Exosomal onco-miRs from serum of patients with adenocarcinoma of the esophagus: comparison of miRNA profiles of exosomes and matching tumor. Tumour biology : the journal of the International Society for Oncodevelopmental Biology and Medicine. 2015; 36: 4643-53. 
34. Lu H, Hao L, Li S, Lin S, Lv L, Chen Y, et al. Elevated circulating stearic acid leads to a major lipotoxic effect on mouse pancreatic beta cells in hyperlipidaemia via a miR-34a-5p-mediated PERK/p53-dependent pathway. Diabetologia. 2016; 59: 1247-57.

35. Gao L, Zhang LJ, Li SH, Wei LL, Luo B, He RQ, et al. Role of miR-452-5p in the tumorigenesis of prostate cancer: A study based on the Cancer Genome Atl(TCGA), Gene Expression Omnibus (GEO), and bioinformatics analysis. Pathology, research and practice. 2018; 214: 732-49.

36. Xiao B, Zhang W, Chen L, Hang J, Wang L, Zhang R, et al. Analysis of the miRNA-mRNA-lncRNA network in human estrogen receptor-positive and estrogen receptor-negative breast cancer based on TCGA data. Gene. 2018; 658: 28-35.

37. Liu Z, Habener JF. Wnt signaling in pancreatic islets. Advances in experimental medicine and biology. 2010; 654: 391-419.

38. Rulifson IC, Karnik SK, Heiser PW, ten Berge D, Chen H, Gu X, et al. Wnt signaling regulates pancreatic beta cell proliferation. Proceedings of the National Academy of Sciences of the United States of America. 2007; 104: 6247-52.

39. Dessimoz J, Bonnard C, Huelsken J, Grapin-Botton A. Pancreas-specific deletion of beta-catenin reveals Wnt-dependent and Wnt-independent functions during development. Current biology : CB. 2005; 15: 1677-83.

40. Grant SF, Thorleifsson G, Reynisdottir I, Benediktsson R, Manolescu A, Sainz $\mathrm{J}$, et al. Variant of transcription factor 7-like 2 (TCF7L2) gene confers risk of type 2 diabetes. Nature genetics. 2006; 38: 320-3.

41. Chen X, Ayala I, Shannon C, Fourcaudot M, Acharya NK, Jenkinson CP, et al. The Diabetes Gene and Wnt Pathway Effector TCF7L2 Regulates Adipocyte Development and Function. Diabetes. 2018; 67: 554-68.

42. Bu L, Yang Q, McMahon L, Xiao GQ, Li F. Wnt suppressor and stem cell regulator TCF7L1 is a sensitive immunohistochemical marker to differentiate testicular seminoma from non-seminomatous germ cell tumor. Experimental and molecular pathology. 2019; 110: 104293.

43. Khan S, Jena GB. Protective role of sodium butyrate, a HDAC inhibitor on beta-cell proliferation, function and glucose homeostasis through modulation of p38/ERK MAPK and apoptotic pathways: study in juvenile diabetic rat. Chemico-biological interactions. 2014; 213: 1-12.

44. Chou PY, Fasman GD. Structural and functional role of leucine residues in proteins. Journal of molecular biology. 1973; 74: 263-81.

45. Siddik MAB, Shin AC. Recent Progress on Branched-Chain Amino Acids in Obesity, Diabetes, and Beyond. Endocrinology and metabolism (Seoul, Korea). 2019; 34: 234-46.

46. Lynch CJ, Adams SH. Branched-chain amino acids in metabolic signalling and insulin resistance. Nature reviews Endocrinology. 2014; 10: 723-36.

47. Ericksen RE, Han W. Malignant manipulaTORs of metabolism: suppressing BCAA catabolism to enhance mTORC1 activity. Molecular \& cellular oncology. 2019; 6: 1585171.

48. White PJ, McGarrah RW, Grimsrud PA, Tso SC, Yang WH, Haldeman JM, et al. The BCKDH Kinase and Phosphatase Integrate BCAA and Lipid Metabolism via Regulation of ATP-Citrate Lyase. Cell metabolism. 2018; 27: 1281-93.e7.

49. Bloomgarden Z. Diabetes and branched-chain amino acids: What is the link? Journal of diabetes. 2018; 10: 350-2.

50. White PJ, Lapworth AL, An J, Wang L, McGarrah RW, Stevens RD, et al Branched-chain amino acid restriction in Zucker-fatty rats improves muscle insulin sensitivity by enhancing efficiency of fatty acid oxidation and acyl-glycine export. Molecular metabolism. 2016; 5: 538-51. 